\title{
Role of Vitamin D as an Adjuvant to Oral Hypoglycemic Drugs in Treatment of Type-2 Diabetic Patients
}

\author{
Sughandha Garg', Debayan Mallik ${ }^{2}$ \\ ${ }^{1}$ Assistant Professor, Department of Pharmacology, Jagannath Gupta Institute of Medical Sciences, ${ }^{2}$ Assistant \\ Professor, Department of Community Medicine, Jagannath Gupta Institute of Medical Sciences
}

Background: Vitamin D deficiency appears to be related to the development of diabetes mellitus type 2 and the metabolic syndrome. Vitamin D may affect glucose homeostasis, vitamin $D$ levels having been found to be inversely related to glycosylated hemoglobin levels in diabetes mellitus. Aims and Objective: The aim of the study was to evaluate role of vitamin $D$ as an adjuvant to oral hypoglycemic drugs in treatment of Type 2 diabetic patients. Materials and Methods: The study was done on 60 patients diagnosed with T2DM. A total of 60 patients, control group were type 2 Diabetic patients on oral hypoglycemic drugs without vitamin $D$ supplementation $(n=30)$ and study group was type 2 Diabetic patients on oral hypoglycemic drugs with vitamin $D$ supplementation $(n=30)$. Results: The mean aged of patients was $43.27 \pm 8.80$ in control group and $47.93 \pm 8.39$ in study group. The family history of diabetes mellitus was 39 patients. HbA1C, FBG, Postprandial were decreased in study group as compared to control group from baseline ( 0 day) to 90 days, respectively. Other parameters like hemoglobin, Serum Creatinine serum glutamic oxaloacetic transaminase and serum glutamic pyruvic transaminase were slight changes in study group as compared to control group from baseline ( 0 day) to 90 days, respectively. Conclusion: The present demonstrated that vitamin $D$ as an adjuvant to oral hypoglycemic drugs in treatment in diabetic patients, vitamin $\mathrm{D}$ levels being related to glycemic control in diabetes mellitus type 2 . These findings may have therapeutic implications as cautious vitamin $D$ supplementation may improve glycemic control in diabetes mellitus type 2 .

Key words: Diabetes mellitus type 2; Glycemic control; Vitamin D

\section{INTRODUCTION}

The World Health Organization defines Diabetes Mellitus as a metabolic disorder characterized by glycosuria, hyperglycemia, hyperlipidemia, negative nitrogen balance and sometimes ketonemia. It is a heterogeneous disorders group having varied etiologies. The pathophysiology involves absolute or relative insulin deficiency along with component of insulin resistance that results from defects in insulin action, insulin secretion or both. The characteristic features include hyperglycemia with altered carbohydrates, proteins and lipid metabolism. The various types of Diabetes according to the eitopathogenesis i.e. Type 1 characterized by complete or near total insulin deficiency and Type 2 involves various degrees of insulin resistance and impaired insulin secretion; Gestational Diabetes: raised blood sugar in $2^{\text {nd }} / 3^{\text {rd }}$ trimester but resolves postpartum; Secondary DM: secondary to other hyperglycemic causes. ${ }^{1}$

Type 2 diabetes mellitus (T2DM) is considered as one of the nonskeletal diseases related with deficiency of vitamin D. Both T2DM and vitamin D deficiency have similar risk factors, such as obesity, aging, and sedentary lifestyle. Cardiovascular diseases (CVDs) and metabolic syndrome disorders are also associated with vitamin $\mathrm{D}$ deficiency. Vitamin D plays an important functional role in glucose homeostasis through its effects on insulin secretion and sensitivity. It may reduce insulin resistance (IR) indirectly 
through its effect on calcium and phosphate metabolism and through upregulation of the insulin receptor gene. ${ }^{2}$ Vitamin D deficiency has been a global pandemic for a while, yet the level of attention given by the scientific and clinical community was only recently stimulated, primarily because of the pleiotropic effects of this hormone outside the skeletal system. Vitamin D deficiency has been consistently associated with hypertension, diabetes mellitus, cardiovascular disease, stroke, multiple sclerosis, inflammatory bowel disease, osteoporosis, periodontal disease, macular degeneration, mental illness, propensity to fall, chronic pain and various cancers. ${ }^{3}$ In addition, vitamin $\mathrm{D}$ deficiency has been described in the metabolic syndrome, ${ }^{4}$ specific vitamin $\mathrm{D}$ receptor gene polymorphisms having been found to be related to components of the metabolic syndrome. Moreover, vitamin D seems to affect glucose homeostasis, vitamin $\mathrm{D}$ levels having been found to be inversely related to glycosylated hemoglobin levels in gestational diabetes mellitus. ${ }^{5}$ However, vitamin D deficiency seems to be related with an increased risk for the development of gestational diabetes mellitus. ${ }^{6}$ We also provided the benefits of vitamin $\mathrm{D}$ supplementation among patients with diabetes mellitus type 2 (DMT2), another chronic non-communicable disease highly prevalent. Previously reported the favourable effects of improved vitamin $\mathrm{D}$ status are most evident in the lipid profile of subjects, reaffirming the hypothesis that vitamin D deficiency contributes to the pathogenesis of atherogenic dyslipidemia. ${ }^{7}$

This purposed of the study was to evaluate role of vitamin $\mathrm{D}$ as an adjuvant to oral hypoglycemic drugs in treatment of Type 2 diabetic patients.

\section{MATERIALS AND METHODS}

This is a study conducted at the primary health care outpatient clinics. The study was done on 60 patients diagnosed with T2DM. A total of 60 patients, control group were 30 and study group was 30 . The patients are divided as following as

- Group A (control group, $\mathrm{n}=30$ ) - Type 2 Diabetic patients on oral hypoglycemic drugs without vitamin D supplementation

- Group B-(study group, $\mathrm{n}=30$ ) - Type 2 Diabetic patients on oral hypoglycemic drugs with vitamin $\mathrm{D}$ supplementation

All the eligible patients were undergone both routine and specific investigations on the first visit. The study group (group B) was prescribed Vitamin D 60,000 IU orally daily for twelve weeks. At the end of the therapy (after 12 weeks) again patients was undergone both routine and specific investigations and was compared from the baseline (day 0).

\section{Inclusion Criteria}

Patients of age more than 30 years but less than 60 years, established diagnosis of Type $2 \mathrm{DM}$ without complications, patients on oral hypoglycaemic drugs and patients without any concurrent illness was included in the study.

\section{Exclusion Criteria}

Patients were excluded if diagnosed as Type $1 \mathrm{DM}$, age less than 30 years as well as more than 60 years, vitamin D supplementation for last 12 weeks, any acute or long term co morbidity and pregnant or lactating women. Necessary laboratory parameters were performed in all patients.

\section{Statistical analysis}

The data was entered in Microsoft excel sheet for analysis and tested statistically on SPSS for windows version 17 software. Quantitative variable were described in descriptive statistical analysis was done for continuous variables, frequency distribution, mean $\pm \mathrm{SD}$ and their percentages for categorical variables were calculated. T-test was used for normal distributed data. $\mathrm{P}$ value $<0.05$ is considered significant.

\section{RESULTS}

A total 60 patients were enrolled in this study; the mean aged of patients was $43.27 \pm 8.80$ in control group and $47.93 \pm 8.39$ in study group (Figure 1). The family history of diabetes mellitus was 39 patients (Figure 2). As shown in Table 1, comparison of mean HbA1C (glycated hemoglobin) before and after 90 days of Vitamin D supplementation showed reduction. With supplementation of Vitamin D mean FBG (fasting blood sugar) values showed a reduction over 90 days period. Postprandial were decreased in study group as compared to control group from baseline (0 day) to 90 days, respectively. Other parameters like hemoglobin, Serum Creatinine serum glutamic oxaloacetic transaminase and serum glutamic pyruvic transaminase were slight changes in study group as compared to control group from baseline ( 0 day) to 90 days, respectively.

\section{DISCUSSION}

This study was design to compare Type 2 Diabetic patients on oral hypoglycemic drugs with and without vitamin $\mathrm{D}$ supplementation. The findings of this study indicate that the metabolic profile of T2DM patients, study group is improved over a period of 90 days as compared to control group, suggesting that vitamin D correction is a promising cardio-protective intervention in vitamin $\mathrm{D}$-deficient populations. The findings of this study indicate that the metabolic profile of T2DM patients, study group 


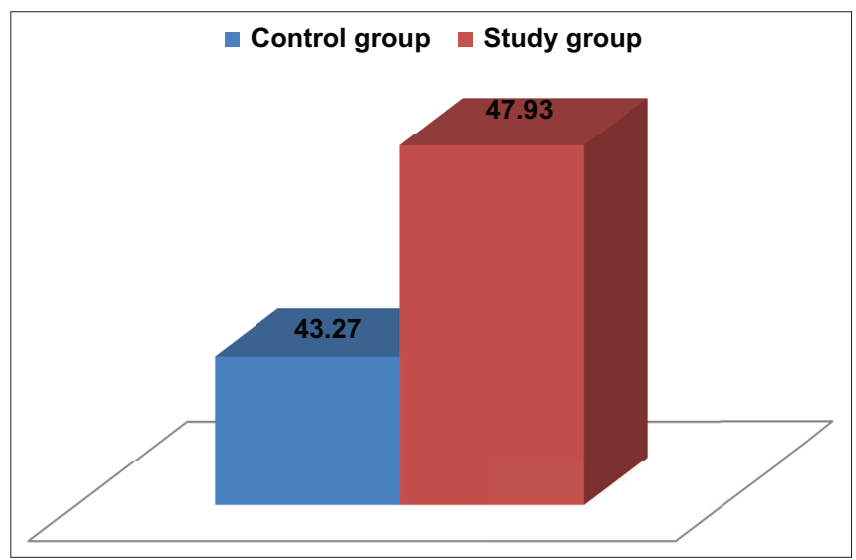

Figure 1: Age of control and study group

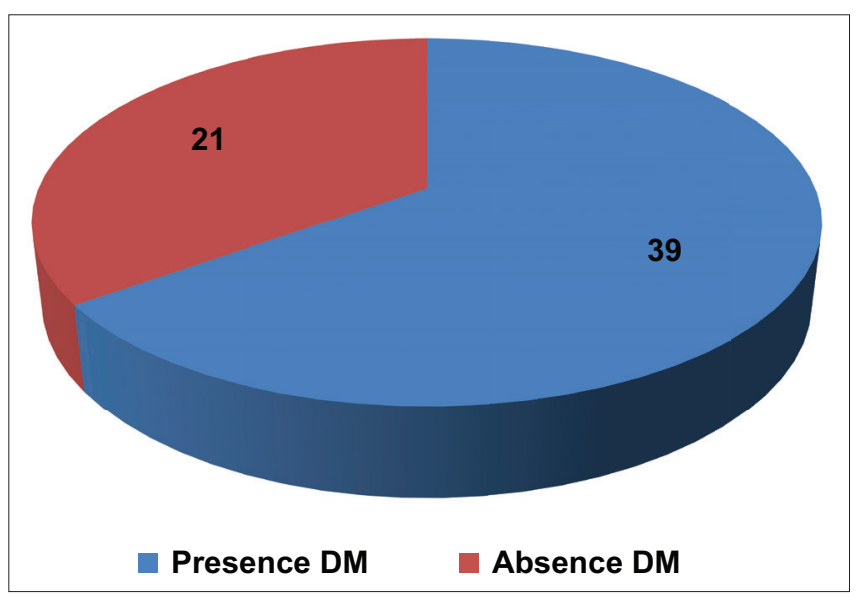

Figure 2: Family history of diabetes mellitus in control and study group

is improved over a period of 90 days as compared to control group, suggesting that vitamin D correction is a promising cardio-protective intervention in vitamin D-deficient populations. A total of 60 patients of Type 2 DM with Vitamin D deficiency not controlled on various oral antidiabetic drugs were included in the study. They were given Vitamin D3 sachet of 60,000 IU weekly for 12 weeks orally and then glycemic status was compared with baseline values. The current study adds to evidence that vitamin D supplementation is most beneficial not only to those who are at high risk patients, but also to those who are deficient and have other extra skeletal chronic diseases, such as diabetes T2DM and cardiovascular disease. Previous study, Eftekhari and colleagues were not able to elicit the same improved metabolic profile in an Iranian T2DM population, which was probably due to a shorter duration of supplementation (12weeks). ${ }^{8}$ Other studies also found no improvement of insulin sensitivity after a high dose vitamin $\mathrm{D}$ intervention. This was probably partly due to the supplementation itself and/or because the subjects were apparently healthy. ${ }^{9}$ Increased insulin resistance post supplementation was observed in a cohort of middle-aged adults, and increased insulin sensitivity in first time GDM

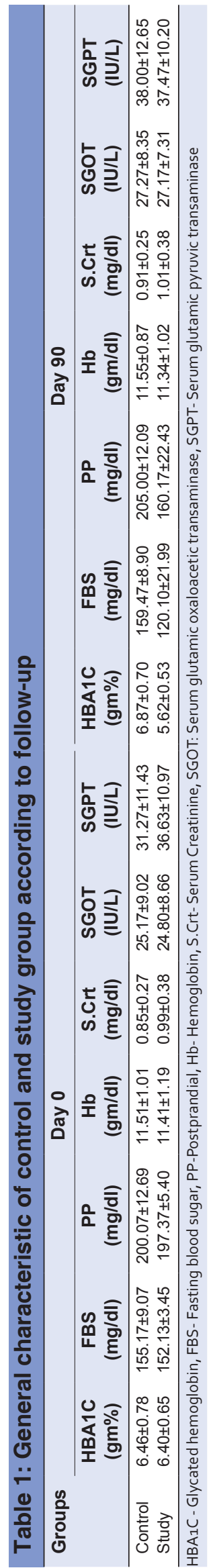


patients. ${ }^{7}$ In the present study, the apparent health benefits were observed after 90 days of supplementation, aside from the expected increase in circulating vitamin $\mathrm{D}$ levels, suggesting that sustained and prolonged supplementation might be necessary to achieve desirable metabolic effects.

Several mechanisms explain how vitamin D can theoretically improve metabolic functions. However, there is enough evidence to support that vitamin D supplementation can independently improve cardiovascular health secondary to its significant associations with cardio metabolic risk factors in both human and animal models, including blood pressure, insulin resistance and aortic media fragmentation, respectively. ${ }^{10}$ The results showed that Vitamin D supplementation of 12 weeks reduces FBG and HbA1C significantly in Vitamin D deficient Type 2 diabetics. Effects of Vitamin D supplementation on glucose homeostasis have been shown by numerous studies. The results of the present study were consistent with many other studies in which FBG and HbA1C improved after Vitamin D supplementation. Also maintain the hemoglobin, serum creatinine, serum glutamic oxaloacetic transaminase, and serum glutamic pyruvic transaminase. Talaei et al. conducted a before and after study on 100 Type 2 DM patients and concluded that there was significant improvement in FBG levels after treatment with 50,000 IU of Vitamin D for 8weeks. ${ }^{11}$ Nasri et al. also concluded that Vitamin D supplementation had beneficial effect on glycemic parameters in Type $2 \mathrm{DM}$ male patients. ${ }^{12}$ Regarding the safety and tolerability of Vitamin D, no untoward side effects or any signs of Vitamin D toxicity were recorded during or after Vitamin D supplementation in the present study, which may be due to the fact that only Vitamin D deficient patients were enrolled in this study and given Vitamin D supplementation for 12 weeks duration.

Several caveats should be mentioned. First, the lack of a placebo-control group and second, the difficulty in the verification of quality of life issues. The study having some limitations included deficient of placebo control group. In addition, the low number of patients and relatively short follow-up period can also be considered limitations.

\section{CONCLUSION}

In conclusion, the study population with results indicating that vitamin $\mathrm{D}$ as an adjuvant to oral hypoglycemic drugs in treatment in diabetic patients. It also indicates the high prevalence of this vitamin deficiency in patients. The findings presented herein have therapeutic implications. In patients with diabetes mellitus type 2, normal levels of vitamin $\mathrm{D}$ in the blood may facilitate glucose control. In addition, in people with a tendency to develop diabetes mellitus type 2, optimal levels of vitamin $\mathrm{D}$ within the blood may retard the clinical development of diabetes mellitus type 2 .

\section{ETHICAL APPROVAL}

The study approved by local ethical committee.

\section{ACKNOWLEDGEMENTS}

Authors would like to thanks all the patients enrolled in this study.

\section{REFERENCES}

1. Diagnosis and Classification of Diabetes Mellitus. Diabetes Care. 2004;27(suppl 1):s5-s10.

2. Nada AM and Shaheen DA. Cholecalciferol improves glycemic control in type 2 diabetic patients: a 6-month prospective interventional study. Therapeutics and clinical risk management 2017; 13:813-820.

3. Alkharfy KM, Al-Daghri NM, Sabico SB, Al-Othman A, Moharram O, Alokail MS, et al. Vitamin D supplementation in patients with diabetes mellitus type 2 on different therapeutic regimens: a oneyear prospective study. Cardiovascular Diabetol 2013;12(1):113.

4. Kayaniyil S, Harris SB, Retnakaran R, Vieth $R$, Knight JA, Gerstein $\mathrm{HC}$, et al. Prospective association of $25(\mathrm{OH}) \mathrm{D}$ with metabolic syndrome. Clinical endocrinol 2014;80(4):502-507.

5. Schuch NJ, Garcia VC, Vivolo SR and Martini LA. Relationship between Vitamin $\mathrm{D}$ Receptor gene polymorphisms and the components of metabolic syndrome. Nutrition journal 2013;12:96.

6. Kostoglou-Athanassiou I, Athanassiou P, Gkountouvas A and Kaldrymides P. Vitamin D and glycemic control in diabetes mellitus type 2. Therapeutic advances in endocrinology and metabolism 2013;4(4):122-128.

7. Al-Daghri NM, Alkharfy KM, Al-Othman A, El-Kholie E, Moharram O, Alokail MS, et al. Vitamin D supplementation as an adjuvant therapy for patients with T2DM: an 18-month prospective interventional study. Cardiovasc Diabetol 2012;11:85.

8. Eftekhari $\mathrm{MH}$, Akbarzadeh $\mathrm{M}$, Dabbaghmanesh $\mathrm{MH}$ and Hasanzadeh J. Impact of treatment with oral calcitriol on glucose indices in type 2 diabetes mellitus patients. Asia Pacific journal of clinical nutrition 2011;20(4):521-526.

9. Grimnes G, Figenschau Y, Almas B and Jorde R. Vitamin D, insulin secretion, sensitivity, and lipids: results from a case-control study and a randomized controlled trial using hyperglycemic clamp technique. Diabetes 2011;60(11):2748-2757.

10. Vacek JL, Vanga SR, Good M, Lai SM, Lakkireddy D and Howard PA. Vitamin D deficiency and supplementation and relation to cardiovascular health. The American journal of cardiol 2012;109(3):359-363.

11. Talaei A, Mohamadi $M$ and Adgi $Z$. The effect of vitamin $D$ on insulin resistance in patients with type 2 diabetes. Diabetology \& metabolic syndrome 2013;5(1):8.

12. Nasri $H$, Behradmanesh $S$, Maghsoudi $A R$, Ahmadi $A$, Nasri $P$ and Rafieian-Kopaei M. Efficacy of supplementary vitamin D on improvement of glycemic parameters in patients with type 2 diabetes mellitus; a randomized double blind clinical trial. Journal of renal injury prevention 2014;3(1):31. 
Authors Contribution:

SG- Concept and design of the study, collected data, reviewed the literature, manuscript preparation and critical revision of the manuscript; DM- Concept, collected data, statistically analyzed and interpreted, review of literature, Manuscript preparation.

Work attributed to:

General Medicine OPD of Rama Medical College Hospital \& Research Centre, Kanpur.

Orcid ID:

Dr. Sughandha Garg- (1) https://orcid.org/0000-0001-5746-8374

Dr. Debayan Mallik- (D) https://orcid.org/0000-0002-1429-5896

Source of Support: Nil, Conflict of Interest: All the authors have no conflict of interest 